\title{
Die Mediterranisierung der Stadtnacht. Das Beispiel Osnabrück
}

\author{
Laura Schäfer ${ }^{1,2}$ \\ Online publiziert: 11. Mai 2020 \\ (c) Der/die Autor(en) 2020
}

\section{Zusammenfassung}

Die Mediterranisierung des öffentlichen Raumes beschreibt die Ausbreitung von Außengastronomie in öffentlichen Räumen sowohl aus räumlicher als auch zeitlicher Perspektive. Diese Entwicklung eröffnet ein neues Themenfeld für die Planungspraxis von Großstädten - die Mediterranisierung der Stadtnacht. Der Beitrag untersucht die Anforderungen der Akteure der Außengastronomie an den öffentlichen Raum, aufbauend auf der Analyse der raumzeitlichen Strukturen sowie Akteursstrukturen am Fallbeispiel Innenstadt Osnabrück. Der Beitrag basiert auf einer Kartierung der Außengastronomie, qualitativen Interviews und einer quantitativen Befragung der Besuchenden der Außengastronomie. Es zeigt sich eine Ballung außengastronomischer Angebote in 2 Quartieren der nördlichen Innenstadt, welche durch unterschiedliche Rahmenbedingungen geprägt sind. Die ermittelten Akteure unterscheiden sich stark in ihrem Handlungsrahmen und im Grad ihrer Vernetzung. Im Ergebnis werden Handlungsfelder für die Planungspraxis ermittelt. Optimierungsbedarf zeigt sich v. a. im Bereich der Verkehrsinfrastruktur und Aufenthaltsqualität sowie in der Kommunikation und strategischen Ausrichtung.

Schlüsselwörter Nachtökonomie $\cdot$ Außengastronomie $\cdot$ Stadtplanung $\cdot$ Öffentlicher Raum $\cdot$ Akteure

\section{The mediterranization of city nights. The example of Osnabrück}

\begin{abstract}
The mediterranization of public space describes the spread of outdoor gastronomy in public spaces from both a spatial and a temporal perspective. This development opens up a new thematic field for the planning practice of large cities-the mediterranization of city nights. This article examines the requirements of the stakeholders of outdoor gastronomy on public space based on the analysis of temporal structures and actor structures, exemplified by the inner city of Osnabrück. The article is based on a mapping of the outdoor gastronomy, qualitative interviews and a quantitative survey of patrons of outdoor gastronomy. There is a concentration of outdoor gastronomic offers in two parts of the northern inner city, which are characterized by different framework conditions. The identified stakeholders differ greatly in their scope of action and in the degree of networking. As a result, fields of action for planning practice are identified. The main need for optimization can be seen in the areas of traffic infrastructure and quality of the stay as well as in communication and strategic orientation.
\end{abstract}

Keywords Nighttime economy $\cdot$ Outdoor gastronomy $\cdot$ Urban planning $\cdot$ Public space $\cdot$ Stakeholders

\section{Außengastronomie als Treffpunkt in der Stadt}

Laura Schäfer, M.A.

laura-schaefer@outlook.de

$1 \quad$ Paulustr. 17, 33602 Bielefeld, Deutschland

2 Universität Osnabrück, Osnabrück, Deutschland
Bereits 1963 widmete sich Jane Jacobs in ihrem Werk „The death and life of great American cities“ (Jacobs 1992) der Bedeutung des öffentlichen Raumes für das urbane Leben. Interessante und attraktive Städte zeichnen sich demnach durch einen belebten Straßenraum mit einer hohen Aufenthaltsqualität aus. Auch der zunehmende Bestand an $\mathrm{Au}$ Bengastronomie im Stadtraum wird in der Literatur dahin- 


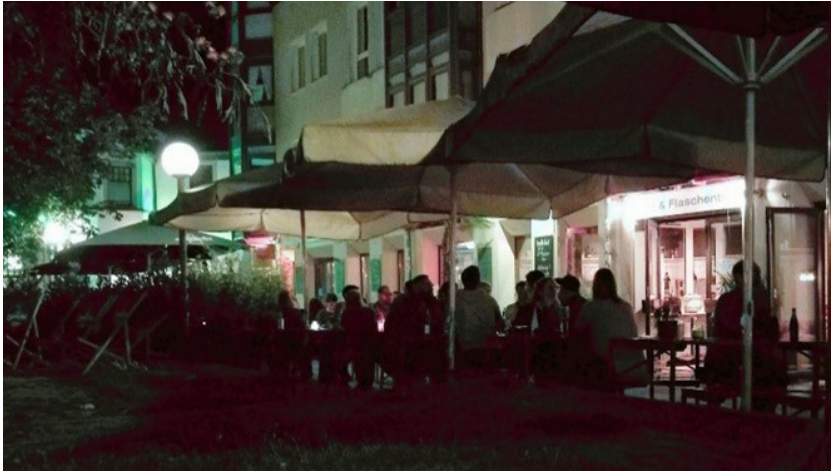

Abb. 1 Außengastronomie am Adolf-Reichwein-Platz in Osnabrïck (eigene Aufnahme: 21.07.2019, 00:46 Uhr)

gehend als Indikator für die Attraktivität einer Stadt angeführt.

Seit geraumer Zeit erfährt der öffentliche Raum nicht zuletzt aufgrund dieser Bedeutungszuschreibung eine zunehmende Ökonomisierung und Privatisierung: Gastronomiebetriebe siedeln sich an und auch das Nachtleben verlagert sich auf die Straße. So sind Straßencafés aus dem heutigen Stadtbild nicht mehr wegzudenken und prägen das urbane Leben (vgl. Junker und Pump-Uhlmann 2019; Abb. 1). Außengastronomie wird so zu einem Treffpunkt in der Stadt. Trotz des großen Einflusses der Gastronomie auf den städtischen Raum ist auch das unter „Mediterranisierung des öffentlichen Raumes" bekannte Phänomen im wissenschaftlichen Diskurs und in der Planungspraxis weitestgehend unbeachtet. Durch Schlagzeilen wie „Mediterranisierung als Knacknuss für Städte“" (Gerny 2012) rücken das Themenfeld und die Bedeutung der Gastronomie für eine Stadt zwar immer weiter in den Fokus des medialen Interesses, dennoch fehlt oftmals die explizite Auseinandersetzung mit dem damit verbundenen Wandel des öffentlichen Raumes. Vor allem der Zeitraum „Nacht“ sollte dabei aufgrund divergierender Interessen zwischen Besuchenden der Außengastronomie und Bewohnerschaft als zwingend notwendiger Planungszeitraum definiert werden (vgl. Schmid 2018).

Aufgrund der vorhandenen Nutzungsmischung innerstädtischer Räume aus Wohnen, Arbeiten, Freizeit und Verkehr ist die Einbindung verschiedenster Gruppen in die Transformationsprozesse notwendig: Neben Gastronomiebetreibenden, Ausgehenden und Anwohnenden sind auch städtische (korporative) Akteure und Mitglieder von Interessensgemeinschaften (kollektive Akteure) wichtige Beteiligte im Zuge städtischer Planungen. Die Ausprägung der Nutzungsstrukturen und die handlungsrelevanten Faktoren der Akteure im Zuge dieser Transformationen im öffentlichen Raum fanden bislang jedoch kaum wissenschaftliche Betrachtung.
„Die Großstadtnacht selbst ist ein Raum-Zeit-Modell, sie will in zeitlichen und in räumlichen Dimensionen ausgemessen und abgeschritten werden“, fordert Schlör (1994, S. 14). Dennoch zeigt sich in der gegenwärtigen Planungspraxis oftmals eine Vernachlässigung des Themas, wie auch Henckel und Kramer 2019 im Hinblick auf den Forschungsgegenstand Zeitgerechtigkeit konstatieren. Der Artikel fokussiert sich dahingehend auf die Ermittlung der raumzeitlichen Strukturen in qualitativer und quantitativer Perspektive. Der Umgang und das Zusammenwirken der einzelnen Beteiligten wird durch äußere Einflüsse, wie klimatische Veränderungen und Veränderungen der Marktwirtschaft, durch Abwägungsbelange wie Märkte- und Zentrenkonzepte sowie durch Zulässigkeiten und Gesetze geprägt. Diese Rahmenbedingungen wirken sich auf unterschiedliche Weise auf die handelnden Akteure aus, welche diese wiederum beeinflussen und gestalten.

Ziel der Forschung ist daher die Aufdeckung gegenwärtiger raumzeitlicher Strukturen im Segment der Außengastronomie am Fallbeispiel des Innenstadtgebiets von Osnabrück sowie die damit einhergehenden Anforderungen der Akteurslandschaft an den Raum. Als Ergebnis werden Handlungsfelder für die Planungspraxis abgeleitet.

Die Forschung kann thematisch in die dritte Welle der Forschung zur Nachtökonomie (vgl. Hadfield 2015) eingeordnet werden, da sie durch die akteursspezifische Betrachtung den Fokus unter anderem auf die Handlungsrahmen der jeweiligen Akteure richtet. Des Weiteren bietet die Arbeit eine wissenschaftliche und empirisch fundierte Auseinandersetzung mit dem Forschungsgegenstand Mediterranisierung. Die Bearbeitung erfolgt anhand des Triangulationsmodells nach Mayring (2008), in welchem die quantitative und die qualitative Forschung parallel verlaufen. Die Analyse basiert auf 8 Leitfadeninterviews, einer Befragung von 221 Besuchenden der Außengastronomie sowie der Kartierung der gastronomischen Angebote im Untersuchungsraum Innenstadt Osnabrück. Der Artikel zeigt im Folgenden die Ergebnisse der empirischen Forschung auf und legt den Fokus zunächst auf die raumzeitlichen Strukturen der Außengastronomie sowie die Akteure der Außengastronomie. Aufbauend auf diesen Erkenntnissen werden Anforderungen der Akteure an Außengastronomie ermittelt und Handlungsempfehlungen abgeleitet.

\section{Raumzeitliche Strukturen der Außengastronomie}

Der Innenstadtbereich der niedersächsischen Studierendenstadt wird durch $43 \%$ Handelsbesatz und $16 \%$ Gastronomiebesatz geprägt (OMT et al. 2019, S. 21). Die Kartierung zeigt einen Gesamtbestand von rund 5000 Sitzplätzen im Untersuchungsraum Osnabrück. 
Abb. 2 Ausprägung der Außengastronomie (eigene Darstellung)

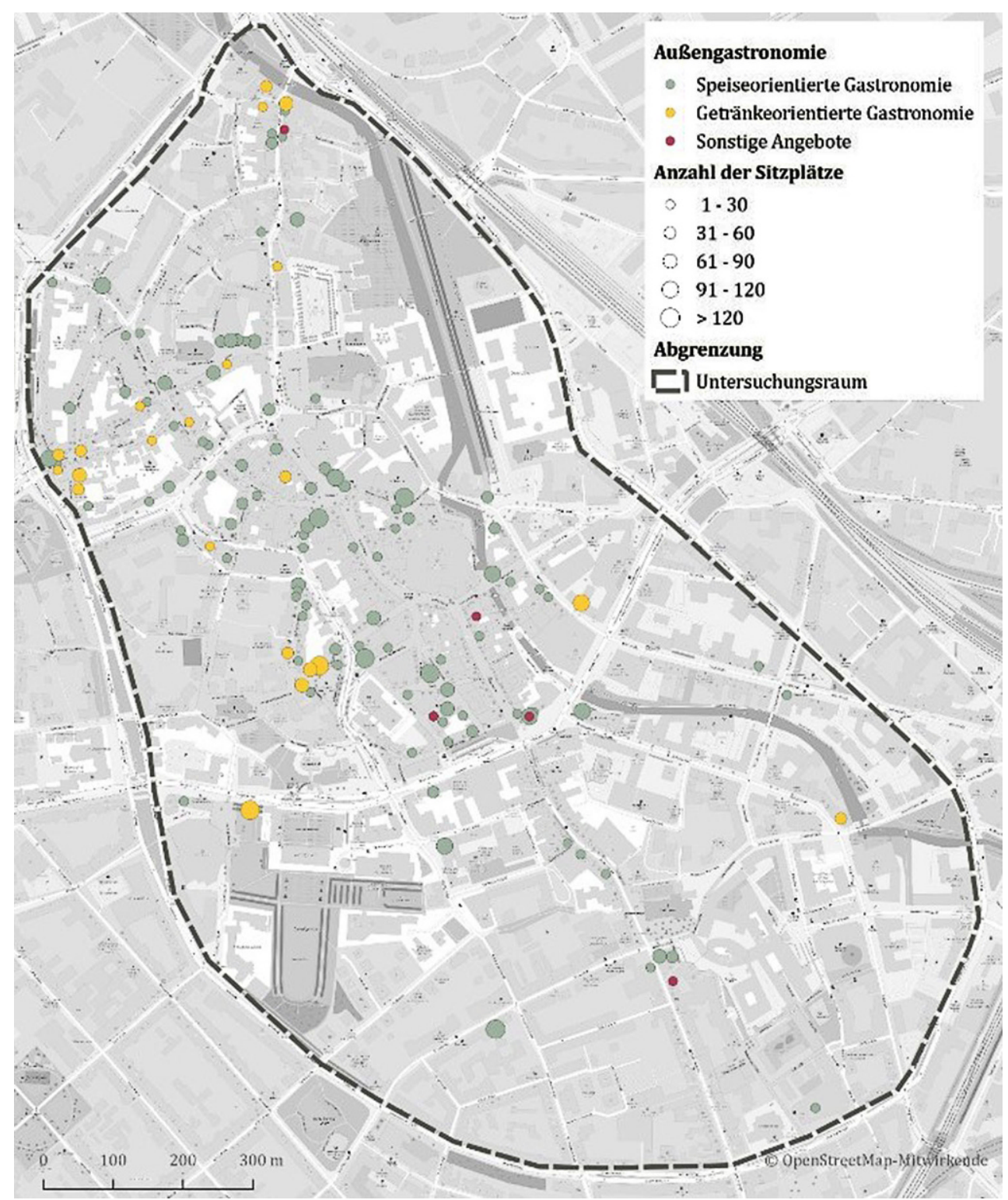

Gastronomiehotspots sowohl in ihrer Ausprägung hinsichtlich der Fläche (Abb. 2) als auch hinsichtlich der Nutzungsintensität (Abb. 3) können in den Quartieren der nördlichen Innenstadt verortet werden. Der meist frequentierte Platz (Adolf-Reichwein-Platz) zeichnet sich durch Individualgastronomie aus (Abb. 1) und wird durch einen Spielplatz ergänzt, gefolgt von einem Platz (Nikolaiort), welcher ausschließlich filialisierte Gastronomie aufweist. Insgesamt lassen sich diverse Gastronomieagglomerationen mit unterschiedlichen Nutzungsprofilen feststellen. Der kurzfristige, zeitliche Nutzungsschwerpunkt liegt an Samstagen und Freitagen in den Abendstunden. Hinsichtlich der langfristigen Nutzung der Außengastronomie kann eine Ausweitung der Sommersaison festgestellt werden. Die nördliche Innenstadt wird durch kulturelle Angebote, Einzelhandel und Gastronomie dominiert. Im Bereich der südlichen Innen- stadt, mit einem höheren Wohnbestand, existieren nur wenige Angebote der Außengastronomie. Die Innenstadt verfügt über ein nahezu flächendeckendes Buslinien- und Parkflächennetz und ist hinsichtlich der Fahrradinfrastruktur gut an die umliegenden Stadtteile angebunden. Die Befragung der ausgehenden Bevölkerung ergab, dass der Untersuchungsraum in Bezug auf seine Raumqualitäten insgesamt sehr positiv bewertet wurde. Identifizierte Schwachpunkte liegen vermehrt im Bereich der Aufenthaltsqualität im öffentlichen Raum sowie in der Verkehrsinfrastruktur.

Im Zuge der Ermittlung raumzeitlicher Strukturen wurde die durch Schmid (2018) geforderte quantitative Datenbasis zur Ausprägung der Außengastronomie geschaffen. Um ein vollständiges Bild der Strukturen sowie der Entwicklungen zu geben, müssten diese Daten in regelmäßigen Abständen im Rahmen eines Monitorings fortlaufend erhoben werden. 
Abb. 3 Nutzungsintensität der Ausgehviertel (eigene Darstellung)

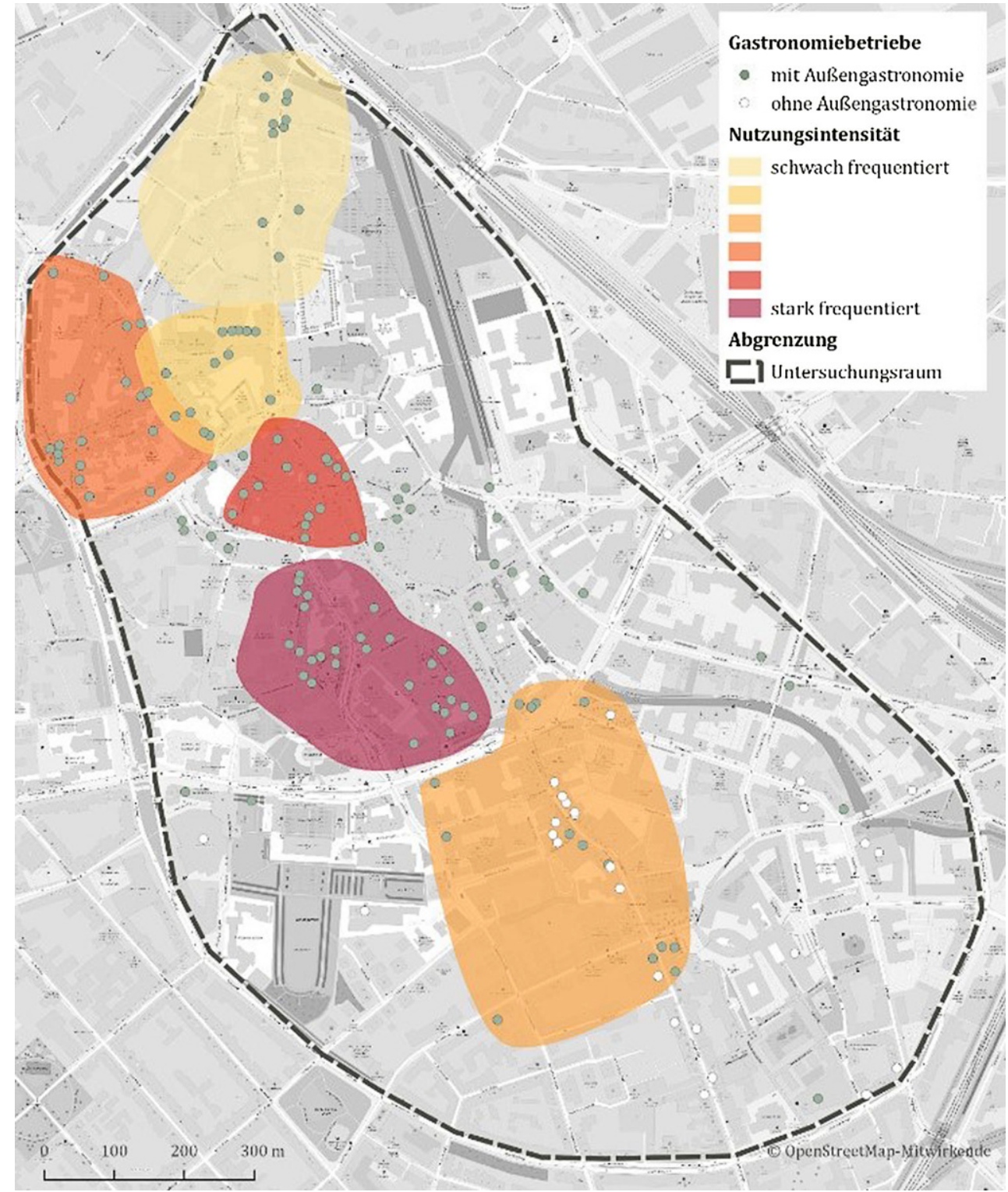

Zudem sind diese um die Wohnraumnutzung in expliziterer Form zu ergänzen.

\section{Akteure der Außengastronomie}

Als zweiter Baustein der Forschung wurden die Akteure der Außengastronomie, ausgehend von korporativen Akteuren, über kollektive Akteure bis hin zu Ausgehenden näher untersucht. Grundlage für die Untersuchung ist der akteurszentrierte Institutionalismus nach Mayntz und Scharpf (1995). Somit wurden u. a. die handlungsrelevanten Faktoren in den Fokus gestellt und Wirkungs- und Spannungsfelder herausgearbeitet.

In den qualitativen Interviews wurde deutlich, dass sich die Akteure in ihrem möglichen Handlungsrahmen stark unterscheiden und es wichtig ist, die jeweilige Rolle zu definieren. Kollektive Akteure in Form von Interessensgemeinschaften und Vereinigungen treten in ihrer Außenwirkung gegenüber der Stadt und bei städtischen Planungen als Multiplikatoren auf und vertreten die kollektiven Interessen in Beteiligungsverfahren. Die ausgehende Bevölkerung, Anwohnenden und Gastronomiebetreibenden sind zunächst als individuelle Akteure zu betrachten, können jedoch oftmals aufgrund jeweils gemeinsamer Interessen zu Gruppen aggregiert werden. Hinsichtlich einer Verlängerung der Öffnungszeiten kann beispielsweise eine Teilgruppe aus den Ausgehenden gebildet werden, während sich Anwohner hinsichtlich einer Eindämmung der Öffnungszeiten zu einer Gruppe formieren können. Im Zuge städtischer Planungen können sie ihre Interessen als Bürgerinnen und Bürger der Stadt einbringen. Sowohl kollektive als 
auch individuelle Akteure haben somit nur die Möglichkeit, auf Themen aufmerksam zu machen bzw. für diese zu sensibilisieren, nicht jedoch übergreifende Entscheidungen bezüglich Planungen zu treffen. ,Aber dann darf die Stadt nicht so affektiert sein, dass man das nicht zulässt, nur, weil man selber nicht draufgekommen ist“, kritisiert ein Vertreter des „Vereins der Osnabrücker Individualgastronomen“. Betreibende eines Gastronomiebetriebs selbst haben zudem die Möglichkeit, auf ihrem eigenen Grundstück im Rahmen der vorhandenen Gesetze und Restriktionen zu handeln. Die Stimmen von individuellen Akteuren können darüber hinaus den medialen Diskurs prägen und sich in Form eines Beteiligungsverfahrens von Bürgerinnen und Bürgern in Märkte- und Zentrenkonzepten und anderen Abwägungsbelangen wiederfinden. Korporative Akteure, welche in der Regel als Top-down-Organisation auftreten und deren Aktivitäten nicht durch individuelle Interessen geprägt sind, verfügen hingegen über Entscheidungsgewalt. Die korporativen Akteure lassen sich zudem in Akteure mit harten Ressourcen u.a. im Bereich Städtebau und Akteure mit ausschließlich weichen Ressourcen wie konzeptionelle Planungen unterscheiden. Dazu sagt der Leiter der Abteilung Stadtentwicklung: ,Wir haben nur die Fähigkeit, Leute zu überzeugen durch Analysen, Informationsgrundlagen zur Verfügung zu stellen, durch eine gemeinsame intellektuelle Beschäftigung damit, andere Sichtweisen vielleicht noch mal einzubringen, die den Tellerrand der Fachplanungen noch mal übersteigen."

Die Auswertung der Interviews zeigt eine Vielzahl divergierender Interessen in Bezug auf den Gegenstand Außengastronomie, woraus Konflikte und Konfliktpotenziale zwischen den Akteuren entstehen können. Diese äußern sich unter anderem in Bezug auf die Ausdehnung der Öffnungszeiten zwischen den Ausgehenden, der Anwohnerschaft, den Gastronomiebetreibenden und dem Ordnungsamt. Die Flächennutzung wirft ein Konfliktpotenzial zwischen den Gastronomiebetreibenden und städtischen Planungen auf, während die Ausgestaltung der Außengastronomie wiederum Konflikte zwischen Gastronomie und Denkmalschutz birgt. Im Hinblick auf die Potenziale städtischer Planungen bleibt zudem als großes Defizit die fehlende strategische Auseinandersetzung festzuhalten, wodurch es keinen expliziten Abwägungsbelang für die gastronomische Entwicklung gibt. Somit besteht keine konzeptionelle Grundlage für den Umgang und die Entwicklung von Außengastronomie und die damit verbundenen Infrastrukturen.

\section{Anforderungen der Akteure an Außengastronomie}

„The working city, the sleeping city and the partying city do not always coexist peacefully“ (Gwiazdzinski 2005,
S. 132 in Henckel und Weber 2019, S. 121). Aus diesem Grund werden unterschiedlichste Anforderungen durch die Akteure an Außengastronomie und den Raum gestellt. Die Befragung der Ausgehenden zeigt den Wunsch nach der Verfügbarkeit von Außengastronomie, um diese sowohl in den Abendstunden als auch anteilig in den Wintermonaten nutzen zu können. So haben insgesamt rund $60 \%$ der befragten Personen ausgedrückt, dass sie explizit ein Lokal mit Außengastronomie aufgesucht haben. Die Erhebung der innerstädtischen Gastronomie zeigt, dass über $75 \%$ der Gastronomiebetreibenden der Anforderung nach Sitzplätzen im Außenbereich nachkommen. Je nach Konzept erfolgt eine Bewirtung auch in den Abend- und Nachtstunden bis 22 Uhr und darüber hinaus, womit sie den Anforderungen der ausgehenden Bevölkerung hinsichtlich der Öffnungszeiten ebenso gerecht werden. In den Wintermonaten reduziert sich in der Regel die Anzahl an Sitzplätzen und wird v. a. für Raucherinnen und Raucher und das äußere Erscheinungsbild der Gastronomie erhalten. Mit der Bereitstellung von Decken wird die Ausdehnung der Nutzungszeiten der AuBengastronomie unterstützt. Handelnde Akteure für diesen Bereich sind in erster Linie die Gastronomiebetreibenden, die auf die Wünsche der Ausgehenden reagieren, als auch das Ordnungsamt, das die Nutzung der öffentlichen Fläche sowie die Nutzungsdauer genehmigt. Sekundär spielen auch die Stadtentwicklung als strategische Planungsinstanz sowie das Stadtmarketing mit der Zielsetzung einer lebendigen Innenstadt eine wichtige Rolle. Gegenwärtig erfolge jedoch keine explizite Auseinandersetzung mit dem Thema Gastronomie, wie der Leiter der Abteilung Stadtentwicklung verdeutlicht: „Wir haben kein Gastronomiekonzept, kein Kneipenkonzept.“

Erreichbarkeit, Sicherheit und Beleuchtung sind 3 Themen die im Rahmen einer zeitgerechten Stadt gemeinsam betrachtet werden sollten (vgl. Henckel und Kramer 2019). Die fußläufige Erreichbarkeit der Gastronomiebetriebe wird ebenfalls positiv wahrgenommen, was sich auch räumlich in der Nähe zu verkehrsfreien Zonen widerspiegelt. Es zeigt sich somit, dass v. a. kleinmaßstäblich für den Fußverkehr ein guter Zugang zur nächtlichen Gastronomielandschaft besteht. Die anderen Verkehrsmittel werden hingegen kritischer betrachtet. Defizitär wird das Angebot des öffentlichen Personennahverkehrs (ÖPNV) eingestuft, welches jedoch flächendeckend mit einer fußläufigen Erreichbarkeit von maximal $200 \mathrm{~m}$ vorliegt. Anforderungen werden an dieser Stelle v. a. durch Ausgehende und ggf. auch Anwohnende gestellt. Vor allem die Infrastruktur für den motorisierten Individualverkehr (MIV), welche im Bereich Heger Tor und Marktplatz ein Defizit für den ruhenden Verkehr aufweist, wird aber negativ beurteilt. Das Gebiet, welches durch verkehrsfreie Zonen und Straßen für Anwohnende geprägt ist und über eine Vielzahl außengastronomischer Angebote verfügt, weist keine großflächigen öffentlichen Park- 


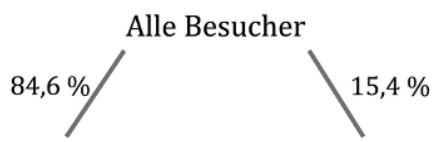

Beleuchtung positiv

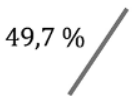

MIV positiv

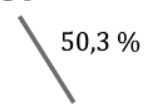

MIV negativ
Beleuchtung negativ

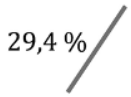

MIV positiv

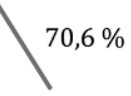

MIV negativ
Abb. 4 Die Wahrnehmung der Beleuchtungssituation in Abhängigkeit zur Einschätzung der Erreichbarkeit durch den MIV, $N=221$ (eigene Erhebung). MIV motorisierter Individualverkehr

platzflächen auf. Die fehlende Ausstattung kann mit den bestehenden Altstadtstrukturen dieser Quartiere begründet werden.

Die Wahrnehmung der Zugänglichkeit der Nacht kann maßgeblich durch die Beleuchtung beeinflusst werden: „The right night-time lighting can make shift workers feel safe on after-dark commutes. It can make you more likely to walk or take public transport. And it can encourage you to socialise outdoors at night, contributing to its nighttime economy.“ (Lam o.J.) Insgesamt werden die Beleuchtungssituation und auch das Sicherheitsempfinden im Untersuchungsraum als überwiegend gut eingeschätzt. Ein Blick auf die Einschätzung der Beleuchtungs- und MIVSituation zeigt jedoch, dass sich abhängig von der Einschätzung der Lichtsituation ein differenziertes Bild über die wahrgenommene MIV-Zugänglichkeit zeigt (Abb. 4).

Neben der MIV- und ÖPNV-Infrastruktur wird auch die hohe Nutzungsmischung zwischen Gastronomie, Fuß- und Fahrradverkehr als potenzielle Gefahrenquelle im Bereich der Gastronomiehotspots identifiziert. Im Sommer 2019 äußerte sich dieses Konfliktpotenzial in einem medial geführten Diskurs um Radabstellanlagen. Dies forderte eine kurzfristige Konfliktlösung zwischen den beteiligten Akteuren, da es anders laut eines städtischen Vertreters einen „kommunikationspolitischen Supergau“ verursacht hätte.

Bezüglich der Auswahlmotive zeigt sich die Atmosphäre innerhalb der Gastronomiebetriebe als positiv konnotiert, das direkte Umfeld wird jedoch selten als ausschlaggebender Grund für den Besuch der Außengastronomie benannt $(34 \%)$. Die positiv wahrgenommenen Kopplungseffekte mit weiteren Nutzungen, wie etwa einkaufen, spiegeln sich in den Strategien des Stadtmarketings wider. Gastronomie wird im Rahmen der Werbemaßnahmen mitgedacht, die ansässigen Gastronomiebetreibenden beteiligen sich jedoch eher selten daran, obwohl diese von der Kopplung profitieren. Die Angebotsvielfalt der Gastronomiebetriebe sowie die Dynamik innerhalb der Gastronomielandschaft werden hinsichtlich einer zunehmenden Filialisierung problematisiert, doch wird hier seitens der korporativen und kollektiven Akteure kein Handlungsspielraum gesehen. Die Aufenthaltsqualität wird hinsichtlich Sauberkeit und Ausgestaltung der Freisitzflächen seitens der ausgehenden Bevölkerung kritisch beäugt und zeigt Optimierungsbedarf. Das Defizit in Bezug auf die Sauberkeit kritisieren gleichzeitig auch städtische und gastronomische Akteure, wobei im Hinblick auf die Ursache auf die ausgehende Bevölkerung verwiesen wird. Die Ausgestaltung der Außengastronomie hingegen wird oftmals durch städtische Rahmenbedingungen, u. a. resultierend aus Richtlinien zum Denkmalschutz in den Altstadtquartieren, beeinflusst. Es zeigt sich,

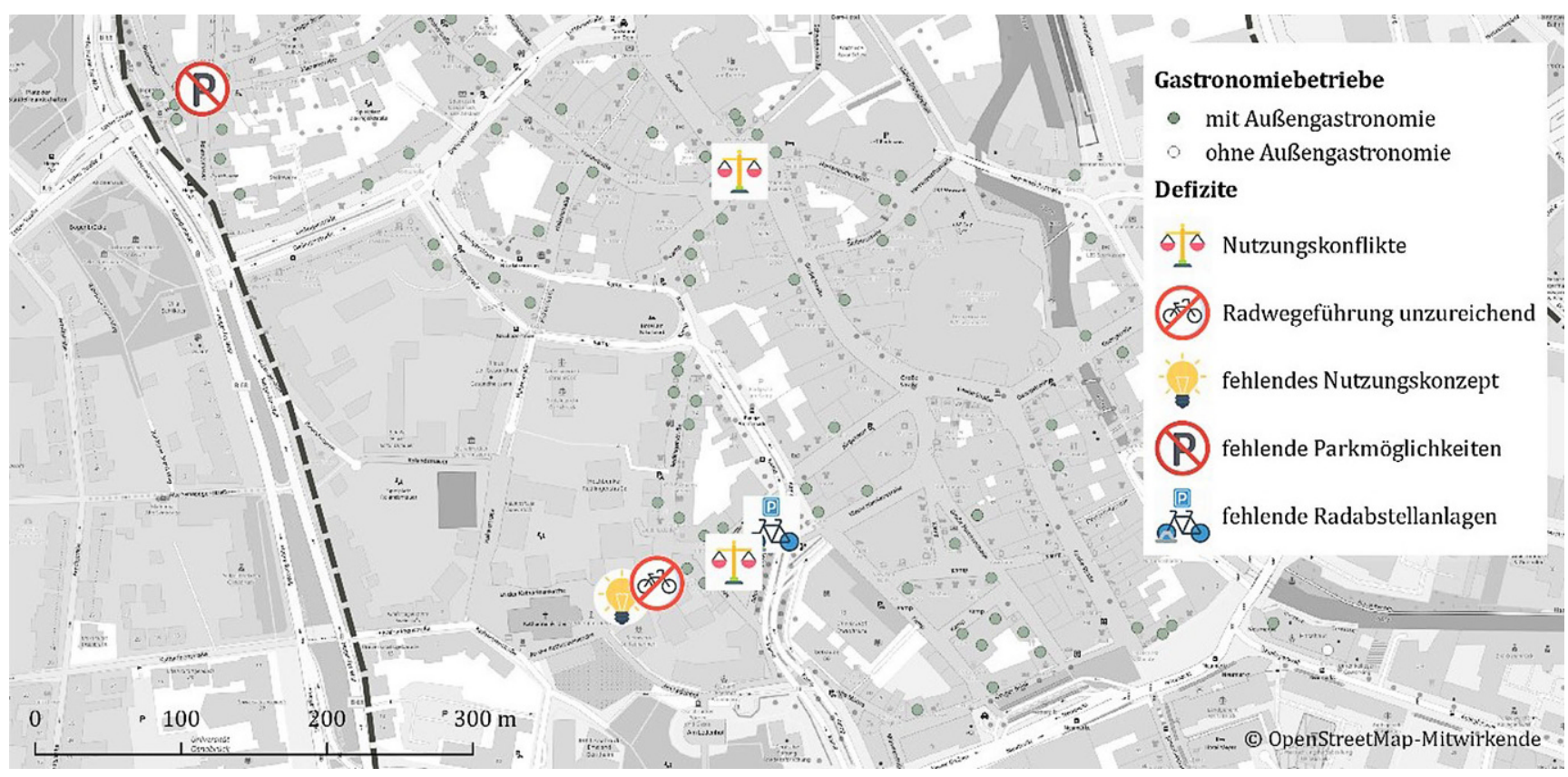

Abb. 5 Verortung der Defizite (eigene Darstellung) 
dass im Besonderen die Ausgestaltung der Außengastronomie im Altstadtbereich zunehmend durch Restriktionen im Bereich Denkmalschutz eingeschränkt wird und eine Reaktion der Gastronomiebetreibenden auf die Wünsche der Besucherinnen und Besucher nur eingeschränkt möglich ist. Auch hier scheint ein Dialog zwischen städtischen Akteuren und Gastronomiebetreibenden zielführend. Weiterhin zeigt sich eine hohe Sitzplatzdichte im Bereich Nikolaiort. Die Ansiedlung weiterer gastronomischer Angebote fordert eine kontinuierliche Auseinandersetzung des Ordnungsamtes mit der Parzellierung der zur Verfügung stehenden Fläche. Die räumlich zu verortenden Defizite sind in Abb. 5 dargestellt.

Die hohe Nutzungsdichte im Untersuchungsraum sowie die unterschiedlichen Anforderungen, die die Bevölkerung an diesen hinsichtlich der Außengastronomie im öffentlichen Raum stellt, bestätigt, dass eine vorausschauende und strategische Auseinandersetzung mit dem Untersuchungsgegenstand notwendig ist (vgl. Schmid 2018; Henckel und Kramer 2019; Eldridge und Roberts 2009). Demnach ist eine weitere Anforderung nicht explizit an den öffentlichen Raum gerichtet, aber dennoch zentral, um den Anforderungen gerecht $\mathrm{zu}$ werden: die Kommunikation im internen Diskurs der handelnden Akteure sowie die nach außen gerichtete Kommunikation. Gegenwärtig positionieren sich die Stadtentwicklung, das Stadtmarketing sowie das Ordnungsamt als vermittelnde Parteien innerhalb der Akteurslandschaft, bestätigen jedoch auch, dass eine Auseinandersetzung meist nicht konzeptionell und vorausschauend, sondern lediglich einzelfall- und bedarfsbezogen vorliegt. Die Rollenzuschreibung wird durch die weiteren Akteure angenommen, jedoch scheinen Kommunikationslücken aufzutreten.

\section{Fazit}

Die Mediterranisierung der Stadtnacht kann für das vorliegende Fallbeispiel Osnabrück belegt werden, jedoch erfolgt oftmals keine explizite Auseinandersetzung mit der Expansion von Außengastronomie. Weiterhin zeigt sich, dass zahlreiche Anforderungen an den öffentlichen Raum und an Außengastronomie erfüllt werden, wie etwa die saisonale und tageszeitliche Ausweitung der Öffnungszeiten und Freisitzflächen.

Dennoch lassen sich diverse Handlungsfelder identifizieren: Die Bereitstellung notwendiger Verkehrsinfrastruktur wird hinsichtlich einer Fußverkehrfreundlichkeit erreicht, der ÖPNV, MIV und auch die Fahrradinfrastruktur bieten jedoch noch Optimierungsspielraum. Neben der Installation weiterer Radabstellanlagen und der Verbesserung der Wegeführung für Fahrräder sollte der Fokus auf die Beleuchtungsstrategien gelegt werden. Der gezielte Einsatz von Leuchtmitteln kann so die Bereitschaft zum Zurücklegen von fußläufigen Distanzen erhöhen. Gleiches setzt sich im Rahmen der Aufenthaltsqualität in Bezug auf die Gestaltung und Sauberkeit fort. Neben der Aufwertung der Stadtmöblierung können verstärkte Grünflächenpflege sowie Sensibilisierungskampagnen dazu beitragen, die Wahrnehmung zu verbessern. Mindergenutzte Flächen können darüber hinaus mit neuen Nutzungskonzepten bespielt und so reaktiviert werden. Weiterhin können Sensibilisierungskampagnen ein gleichberechtigtes Nebeneinander unterschiedlicher Nutzungen fördern.

Für eine vollständige Betrachtung müssen die Anforderungen aus raumzeitlicher Perspektive durch Anforderungen aus akteurszentrierter Perspektive ergänzt werden (vgl. Schmid 2018). Zum einen bezieht sich das auf die Котmиnikation, da durch einen frühzeitigen Austausch der Beteiligten und durch die Erarbeitung von Strategien zum Umgang mit der Mediterranisierung der Stadtnacht Konflikte, Zuständigkeitsprobleme und Fehlplanungen minimiert werden können. Um die Kommunikation zu erleichtern, wird die Initiierung eines Trialoges vorgeschlagen: „Vielleicht muss man da eher einen Trialog organisieren zwischen Nutzern, Anbietern und Anwohnern.“ (Interview Abteilungsleitung Stadtentwicklung) Die öffentliche Kommunikation, beispielsweise im medialen Diskurs, kann zudem dazu beitragen, für bestimmte Entwicklungen zu sensibilisieren und somit Akzeptanz und Zufriedenheit innerhalb der Akteurslandschaft und der Bevölkerung zu schaffen. Zum anderen sollte ein gezielter Einsatz personeller und finanzieller Kapazitäten zur Entwicklung und Umsetzung von Strategien erfolgen. Im Untersuchungsraum besteht gegenwärtig kein Konzept zur gastronomischen Entwicklung, obwohl die Gastronomie - nicht zuletzt aufgrund der Studierenden in der Stadt als auch aufgrund der Rolle als Oberzentrum der Region - als wichtiger Standortfaktor herangeführt werden kann. Oberstes Ziel der korporativen Akteure sollte es daher sein, auf gesamtstädtischer Ebene Strategien zum Umgang mit Gastronomie - u. a. mit Fokus auf die genannten Handlungsfelder Kommunikation, Verkehrsinfrastruktur und Aufenthaltsqualität - zu entwickeln.

Der vorliegende Artikel dient als Impuls für eine dezidierte Behandlung der Außengastronomie als Thema in Wissenschaft und Praxis. Aufgrund des Forschungsdesigns der Fallstudie sind die Ergebnisse nur bedingt auf andere Städte übertragbar, können jedoch als Denkanstoß für Akteure anderer Kommunen dienen, ermittelte Defizite zu überprüfen und Außengastronomie als Baustein für eine Belebung der Innenstadt zu betrachten.

Funding Open Access funding provided by Projekt DEAL.

Open Access Dieser Artikel wird unter der Creative Commons Namensnennung 4.0 International Lizenz veröffentlicht, welche die Nutzung, Vervielfältigung, Bearbeitung, Verbreitung und Wiedergabe in 
jeglichem Medium und Format erlaubt, sofern Sie den/die ursprünglichen Autor(en) und die Quelle ordnungsgemäß nennen, einen Link zur Creative Commons Lizenz beifügen und angeben, ob Änderungen vorgenommen wurden.

Die in diesem Artikel enthaltenen Bilder und sonstiges Drittmaterial unterliegen ebenfalls der genannten Creative Commons Lizenz, sofern sich aus der Abbildungslegende nichts anderes ergibt. Sofern das betreffende Material nicht unter der genannten Creative Commons Lizenz steht und die betreffende Handlung nicht nach gesetzlichen Vorschriften erlaubt ist, ist für die oben aufgeführten Weiterverwendungen des Materials die Einwilligung des jeweiligen Rechteinhabers einzuholen.

Weitere Details zur Lizenz entnehmen Sie bitte der Lizenzinformation auf http://creativecommons.org/licenses/by/4.0/deed.de.

\section{Literatur}

Eldridge A, Roberts M (2009) Planning the night-time city. Taylor and Francis, Hoboken

Gerny D (2012) Mediterranisierung als Knacknuss für Städte: Neue Züricher Zeitung. https://www.nzz.ch/meinung/kommentare/ die-mediterranisierung-als-neue-knacknuss-fuer-die-staedte- 1 . 17174229. Zugegriffen: 2. Apr. 2019

Hadfield P (2015) The night-time city. Four modes of exclusion: reflections on the Urban studies special collection. Urban Stud 52(3):606-616

Henckel D, Kramer C (Hrsg) (2019) Zeitgerechte Stadt. Konzepte und Perspektiven für die Planungspraxis. Forschungsberichte der ARL, 09. Akademie für Raumforschung und Landesplanung Leibniz-Forum für Raumwissenschaften, Hannover

Jacobs J (1992) The death and life of great American cities. Vintage Books, New York

Junker R, Pump-Uhlmann H (2019) Einkaufsstraßen neu denken Bausteine für neue Perspektiven. Landesinitiative StadtBauKultur NRW, Gelsenkirchen

Lam F (2019) Cities alive-lighting the urban night time. Arup. https:// www.arup.com/perspectives/cities-alive-lighting-the-urban-nighttime. Zugegriffen: 22. Nov. 2019

Mayntz R, Scharpf FW (Hrsg) (1995) Gesellschaftliche Selbstregelung und politische Steuerung. Schriften des Max-Planck-Instituts für Gesellschaftsforschung Köln, 23. Campus, Frankfurt am Main

Mayring P (2008) Qualitative Inhaltsanalyse. In: Mayring P, Gläser-Zikuda M (Hrsg) Die Praxis der Qualitativen Inhaltsanalyse. Beltz, Weinheim

Osnabrück-Marketing und Tourismus GmbH (OMT), Osnabrücker City Marketing e. V. (OCM), Industrie- und Handelskammer Osnabrück - Emsland - Grafschaft Bentheim (IHK) (2019) Handelsmonitor Osnabrück 2019. https://www.osnabrueck.de/fileadmin/ user_upload/Handelsmonitor2019_Gesamtbericht_20190529. pdf. Zugegriffen: 1. Okt. 2019

Schlör J (1994) Nachts in der großen Stadt. Paris, Berlin, London 1840-1930. dtv, 30428. Dt.Taschenbuch Verl, München

Schmid JF (2018) stadtnachacht - Stadtentwicklungspolitische Instrumente für das Management der urbanen Nachtökonomie. Dissertation. HafenCity Universität Hamburg, Hamburg 